\title{
Cognitive functions of the posterior parietal cortex
}

\author{
Christos Constantinidis ${ }^{1}$, David J. Bucci ${ }^{2}$ and Michael D. Rugg ${ }^{3}$ \\ Department of Neurobiology and Anatomy, Wake Forest School of Medicine, Winston-Salem, NC, USA \\ 2 Psychological and Brain Sciences, Dartmouth College, Hanover, NH, USA \\ ${ }^{3}$ Center for Vital Longevity and School of Behavioral and Brain Sciences, University of Texas at Dallas, Dallas, TX, USA \\ *Correspondence: cconstan@wfubmc.edu
}

Edited by:

Sidney A. Simon, Duke University, USA

The posterior parietal cortex has traditionally been associated with visuo-spatial perception and spatial attention, however, accumulating evidence indicates that it involved in a much wider range of cognitive functions. The articles included in the E-book review experimental data and theoretical considerations, as well as reviews of recent work supporting this idea. Anatomical, lesion, neurophysiological, and functional imaging data are discussed. Animal models (rodent and primate) as well as human studies are covered. Finally, the unique and shared functions of the posterior parietal cortex are compared to other brain areas. These contributions provide a primer of the current state of knowledge, identify unresolved questions, highlight recent conceptual and methodological advances, and, we hope, will stimulate future research.

In the first part of the E-book, evidence from rodent model systems is presented. Articles examine the contribution of animal models to long-term memory (Myskiw and Izquierdo, 2012), illusory conjunctions (Kesner, 2012), ranking of topographic signals (Broussard, 2012), and relational learning (Robinson and Bucci, 2012). A common theme across these topics is the intersection of attentional functions of posterior parietal cortex with learning/memory-related processes. Data are presented from studies that combine experimental lesion techniques and electrophysiological methods with sophisticated behavioral assays that attempt to elucidate the precise contributions of posterior parietal cortex.

A series of experiments in non-human primate models similarly reveal activation of the posterior parietal cortex in a variety of cognitive functions, such as numerosity (Roitman et al., 2012), categorization (Fitzgerald et al., 2012), and decision-making (Huk and Meister, 2012). Spatial signals are present and shape

\section{REFERENCES}

Berryhill, M. E. (2012). Insights from neuropsychology: pinpointing the role of the posterior parietal cortex in episodic and working memory. Front. Integr. Neurosci. 6:31. doi: 10.3389/fnint.2012.00031

Broussard, J. I. (2012). Posterior parietal cortex dynamically ranks topographic signals via cholinergic influence. Front. Integr. Neurosci. 6:32. doi: 10.3389/fnint.2012.00032

Chafee, M. V., and Crowe, D. A. (2012). Thinking in spatial terms: decoupling spatial representation from sensorimotor control in monkey posterior parietal areas $7 \mathrm{a}$ and LIP. Front. Integr. Neurosci. 6:112. doi: 10.3389/fnint.2012.00112

Fitzgerald, J. K., Swaminathan, S. K., and Freedman, D. J. (2012). Visual categorization and the parietal cortex. Front. Integr. Neurosci. 6:18. doi: 10.3389/fnint.2012.00018

Hadjidimitrakis, K., Breveglieri, R., Bosco, A., and Fattori, P. (2012). Three-dimensional eye position signals shape both peripersonal space

peri-personal shape and limb movements (Hadjidimitrakis et al., 2012), however, spatial information also forms an abstract spatial representation that can be decoupled from sensorimotor control (Chafee and Crowe, 2012). Neurophysiological experiments provide insights on the nature of differences between the posterior parietal cortex and other cortical areas, such as the prefrontal cortex, in the context of visual search (Wardak et al., 2012) and other tasks (Katsuki and Constantinidis, 2012). The conclusion that emerges from these studies is that the posterior parietal cortex is activated in a wide range of tasks, and individual parietal neurons exhibit neural correlates of complex cognitive functions.

In the last part of the E-book, evidence from human studies is considered. Imaging studies routinely reveal BOLD activation during episodic memory tasks (Berryhill, 2012; Levy, 2012). In recent years, nuanced memory deficits following parietal lesions have also been recognized (Berryhill, 2012). EEG and MEG studies have yielded consistent evidence about the time course of parietal mnemonic activation (Levy, 2012). Both process- and content-based models have been proposed to account for the nature of this activation (Berryhill, 2012; Levy, 2012). Finally, the posterior parietal cortex has been implicated in cognitive control, with different subdivisions proposed to be specialized for bottom-up and top-down control (Shomstein, 2012).

Collectively, these studies illustrate our current understanding of the posterior parietal cortex with regard to cognitive operations. While the nature and extent of its involvement continues to be investigated, it is now clear that its role goes beyond the functions traditionally ascribed to it, spatial representation and attention—a major development of the past few years.

and arm movement activity in the medial posterior parietal cortex. Front. Integr. Neurosci. 6:37. doi: 10.3389/fnint.2012.00037

Huk, A. C., and Meister, M. L. (2012). Neural correlates and neural computations in posterior parietal cortex during perceptual decisionmaking. Front. Integr. Neurosci. 6:86. doi: 10.3389/fnint.2012.00086

Katsuki, F., and Constantinidis, C. (2012). Unique and shared roles of the posterior parietal and dorsolateral prefrontal cortex in cognitive functions. Front. Integr. Neurosci. 6:17. doi: 10.3389/fnint.2012.00017

Kesner, R. P. (2012). Parietal lesions produce illusory conjunction errors in rats. Front. Integr. Neurosci. 6:22. doi: 10.3389/fnint.2012.00022

Levy, D. A. (2012). Towards an understanding of parietal mnemonic processes: some conceptual guideposts. Front. Integr. Neurosci. 6:41. doi: 10.3389/fnint.2012.00041

Myskiw, J. C., and Izquierdo, I. (2012). Posterior parietal cortex and long-term memory: 
some data from laboratory animals. Front. Integr. Neurosci. 6:8. doi: 10.3389/fnint.2012.00008

Robinson, S., and Bucci, D. J. (2012). Damage to posterior parietal cortex impairs two forms of relational learning. Front. Integr. Neurosci. 6:45. doi: 10.3389/fnint.2012.00045

Roitman, J. D., Brannon, E. M., and Platt, M. L. (2012). Representation of numerosity in posterior parietal cortex. Front. Integr. Neurosci. 6:25. doi: 10.3389/fnint.2012.00025

Shomstein, S. (2012). Cognitive functions of the posterior parietal cortex: top-down and bottom-up attentional control. Front. Integr. Neurosci. 6:38. doi: 10.3389/fnint.2012.00038

Wardak, C., Ben Hamed, S., Olivier, E., and Duhamel, J. R. (2012). Differential effects of parietal and frontal inactivations on reaction times distributions in a visual search task. Front. Integr. Neurosci. 6:39. doi: 10.3389/fnint.2012.00039

Received: 02 April 2013; accepted: 23 April 2013; published online: 09 May 2013.

Citation: Constantinidis C, Bucci DJ and Rugg MD (2013) Cognitive functions of the posterior parietal cortex. Front.
Integr. Neurosci. 7:35. doi: 10.3389/fnint. 2013.00035

Copyright (C) 2013 Constantinidis, Bucci and Rugg. This is an open-access article distributed under the terms of the Creative Commons Attribution License, which permits use, distribution and reproduction in other forums, provided the original authors and source are credited and subject to any copyright notices concerning any third-party graphics etc. 